\title{
Exploitation of Heterosis in Inter-Varietal Crosses of Urdbean (Vigna mungo [L.] Hepper)
}

\author{
A.V.S. Durga Prasad ${ }^{1 *}$ and E. Murugan ${ }^{2}$ \\ ${ }^{1}$ Department of Genetics and Plant Breeding, RARS, Maruteru - 534 122, India \\ ${ }^{2}$ Department of Genetics and Plant Breeding, AC\& RI, Madurai - 625 104, India \\ *Corresponding author
}

\section{Keywords}

Line $\mathrm{x}$ Tester, ANOVA, Heterosis and Seedyield, Plant

\section{Article Info}

Accepted:

12 December 2020

Available Online:

10 January 2021

\section{A B S T R A C T}

Field trial was conducted to assess the magnitude of heterosis for nine traits in 33 inter-varietal crosses (synthesized by crossing 11 lines and 3 testers in LxT fashion) of urdbean along with a check variety ' $\mathrm{VBN}(\mathrm{Bg}) 4$ ' towards exploitation of hybrid vigour for early maturity and higher yields. The ANOVA revealed that mean squares due to genotypes, parents, crosses, lines, testers (except for plant height) and lines $x$ testers (except plant height) were highly significant for all the traits under evaluation connoting the existence of wider genetic diversity among them. In general, the inter-varietal crosses showed desired heterosis for days to 50 per cent flowering and plant height i.e., negatively significant relative heterosis, heterobeltiosis and standard heterosis indicating their earliness in maturity. Based on experimental results of this investigation, two inter-varietal crosses viz., LBG 709 x PU 31, LBG 645 x VBN (Bg)6 and LBG 645 x PU 31 were found promising for most of the traits including seed yield. Therefore, exploitation of hybrid vigour may be rewarding in these crosses, paving way for genetic enhancement of this crop.

\section{Introduction}

Urdbean $(2 \mathrm{n}=22)$, popularly known as blackgram or mash is a premier short duration legume crop belonging to family leguminosae. It is an excellent source of high quality easily digestible dietary protein (25$28 \%)$, oil (1.0-1.5\%), fiber (3.5\%-4.5\%, ash (4.5-5.5\%), carbohydrates (62-65\%), low flatulence, fairly supplier of lysine, vitamins iron and phosphorus. Being a short duration legume and photo-thermo insensitive nature, it is considered as excellent for crop intensification and diversification. Besides like any other legume, this crop acts as an ameliorator of soil fertility through symbiotic nitrogen fixation as well as a green manure. However in recent years, lack of promising 
genotypes suited to local environments is a major constraint hindering its production, necessitating the development of new varieties adapted to local condition. Extent and magnitude of heterosis present in hybrids is vital aspect for any crop improvement programme. The presence of heterosis in food legumes has also been demonstrated by Kant and Srivastava (2012). Little information about heterosis and gene action is available in blackgram. The exploitation of heterosis in urdbean has not been commercialized due to limited extent of out crossing (Singh, 2000). However, highly heterotic crosses can be used for development of high yielding pure line varieties in a self-pollinated crop like urdbean. Hence, the present study was undertaken to assess the magnitude of heterosis in order to harness the hybrid vigour of inter-varietal crosses in urdbean.

\section{Materials and Methods}

Fourteen varieties / cultures of urdbean adapted to various agro-climatic condition were crossed in Line $\mathrm{x}$ Tester fashion which comprised of eleven lines viz., ACM 05007, MDU 1, ADT 3, Co 5, Co 6, LBG 623, LBG 645, LBG 685, LBG 709, TMV 1 and VBN (Bg) 5 and three testers viz., PU 31, VBN (Bg) 4 and VBN (Bg) 6 during October, 2013 at Agricultural College and Research Institute, Madurai to obtain 33 inter-varietal crosses. The resultant 33 hybrids were raised along with their respective parents in RBD, replicated twice with a spacing of $30 \times 10 \mathrm{~cm}$ at National Pulses Research Centre, Vamban, during July, 2014. Each plot consisted of $4 \mathrm{~m}$ row length with a spacing of $30 \times 10 \mathrm{~cm}$ between row and plants, respectively. Data were recorded on 10 randomly selected in each replication for nine quantitative traits viz., days to $50 \%$ flowering, plant height, number of branches / plant, number of clusters / plant, number of pods / plant, number of seeds / pod, hundred seed weight and seed yield / plant. The mean data on above traits were used to compute relative heterosis, heterobeltiosis, and standard heterosis (Hays et al., 1955). Variety 'VBN (Bg)4' was used as the standard parent, as it is one of the best released variety in Tamil Nadu. The mean values of hybrids and their respective parents were used for estimation of different heterosis per cent under three categories based on the formula suggested by Fonseca and Patterson, (1968) the test of significance for estimates of heterosis were tested for significance at error degrees of freedom as suggested by Turner (1953).

\section{Results and Discussion}

Analysis of variance (ANOVA) for LxT analysis in urdbean for yield and its attributes were computed (Table 1). The ANOVA revealed that mean squares due to genotypes, parents, crosses, lines, testers (except for plant height) and lines $\mathrm{x}$ testers (except plant height) were highly significant for all the traits under evaluation connoting the existence of wider genetic diversity among them This justified the selection of 14 genotypes as parents in the present $\mathrm{L} \times \mathrm{T}$ analysis. The results of heterosis over mid parent, better parent and check variety i.e., VBN (Bg)4 were presented in Tables 2, 3 and 4. Among three estimates of heterosis, in general heterosis over commercial check has been widely considered as important estimate of heterosis for commercial exploitation of hybrid vigour of crosses (Grakh and Chaudhury, 1985).

The extent of heterosis depends on the magnitude of nonadditive gene action and wide genetic diversity among parents (Ram et al., 2013). For the trait, days to $50 \%$ flowering, the relative heterosis ranged from 5.71 to 5.51 and 13 inter-varietal crosses displayed desired significant and negative standard heterosis. 
Table.1 Analysis of variance for LxT analysis in urdbean for yield and its attributes

\begin{tabular}{|c|c|c|c|c|c|c|c|c|c|c|}
\hline \multirow[t]{2}{*}{ Source } & \multirow[t]{2}{*}{ df } & \multicolumn{9}{|c|}{ Mean Squares } \\
\hline & & $\begin{array}{c}\text { Days to } 50 \% \\
\text { flowering }\end{array}$ & $\begin{array}{l}\text { Plant } \\
\text { height }\end{array}$ & $\begin{array}{c}\text { Number of } \\
\text { branches/plant }\end{array}$ & $\begin{array}{c}\text { Number of } \\
\text { clusters/plant }\end{array}$ & $\begin{array}{l}\text { Number of } \\
\text { pods/plant }\end{array}$ & $\begin{array}{l}\text { Pod } \\
\text { length }\end{array}$ & $\begin{array}{l}\text { Number of } \\
\text { seeds/pod }\end{array}$ & $\begin{array}{c}100 \text { seed } \\
\text { weight }\end{array}$ & Seed yield/plant \\
\hline Replication & 1 & 0.89 & 0.05 & 0.03 & 0.04 & 0.01 & 0.01 & 0.09 & 0.03 & 0.01 \\
\hline Genotypes & 46 & $5.03 *$ & $57.58 *$ & $0.15^{*}$ & $18.42 *$ & $36.80 *$ & $0.08 *$ & $0.26^{*}$ & $0.28 *$ & $6.52 *$ \\
\hline Parents & 13 & $8.09 *$ & $128.93 *$ & $0.13 *$ & $26.31 *$ & $20.57 *$ & $0.13 *$ & $0.23 *$ & $0.52 *$ & $4.44 *$ \\
\hline Crosses & 32 & $3.94 *$ & $15.28 *$ & $0.16^{*}$ & $13.65 *$ & $36.63 *$ & $0.07 *$ & $0.23 *$ & $0.17 *$ & $5.47 *$ \\
\hline Parents Vs Crosses & 1 & $0.12 *$ & $483.69 *$ & $0.07 *$ & $68.55^{*}$ & $253.66^{*}$ & $0.03 *$ & $1.72 *$ & $0.75^{*}$ & $67.31 *$ \\
\hline Lines & 10 & $6.63 *$ & $35.53 *$ & $0.15^{*}$ & $22.92 *$ & $86.58 *$ & $0.12 *$ & $0.42 *$ & $0.37 *$ & $12.38 *$ \\
\hline Testers & 2 & $21.02 *$ & 2.47 & $0.50 *$ & $11.02 *$ & $47.72 *$ & $0.04 *$ & $0.22 *$ & $0.17 *$ & $2.67 *$ \\
\hline Lines $x$ Testers & 20 & $0.88 *$ & 6.44 & $0.13 *$ & $9.28 *$ & $10.54 *$ & $0.04 *$ & $0.13 *$ & $0.07 *$ & $2.29 *$ \\
\hline Error & 46 & 0.12 & 3.23 & 0.01 & 0.40 & 2.60 & 0.01 & 0.02 & 0.04 & 0.23 \\
\hline
\end{tabular}

* Significant at $5 \%$ level 
Table.2 Heterosis percent for Days to 50\% flowering, Plant height and Number of branches/plant

\begin{tabular}{|c|c|c|c|c|c|c|c|c|c|}
\hline \multirow[t]{2}{*}{ Crosses } & \multicolumn{3}{|c|}{ Days to $50 \%$ flowering } & \multicolumn{3}{|c|}{ Plant height } & \multicolumn{3}{|c|}{ Number of branches/plant } \\
\hline & $\mathbf{d}_{\mathrm{i}}$ & $\mathbf{d}_{\mathrm{ii}}$ & $\mathbf{d}_{\mathrm{iii}}$ & $\mathbf{d}_{\mathrm{i}}$ & $\mathbf{d}_{\mathrm{ii}}$ & $\mathbf{d}_{\mathrm{iii}}$ & $\mathbf{d}_{\mathrm{i}}$ & $\mathbf{d}_{\mathrm{ii}}$ & $\mathbf{d}_{\mathrm{iii}}$ \\
\hline ACM 05007 x PU 31 & 0.00 & -3.08 & $-8.70 * *$ & -0.82 & $-13.85 * *$ & 4.96 & 4.90 & 2.88 & $-18.94 * *$ \\
\hline ACM 05007 x VBN (Bg)4 & 1.49 & -1.45 & -1.45 & $-12.53 * *$ & $-20.37 * *$ & -2.98 & $-10.34 * *$ & $-19.85 * *$ & $-19.85 * *$ \\
\hline ACM 05007 x VBN(Bg) 6 & -3.82 & -4.55 & $-8.70 * *$ & -3.37 & $-32.89 * *$ & $-18.24 * *$ & -4.47 & -5.38 & $-25.45^{* *}$ \\
\hline MDU 1 x PU 31 & $5.51 *$ & 1.52 & -2.90 & $-16.06 * *$ & $-28.24 * *$ & $-9.18^{*}$ & $7.41 *$ & 0.00 & $-12.12 * *$ \\
\hline MDU 1x VBN (Bg)4 & -2.22 & -4.35 & -4.35 & $-23.33 * *$ & $-31.37 * *$ & $-13.15 * *$ & $-10.16 * *$ & $-15.61 * *$ & $-15.61 * *$ \\
\hline MDU 1 x VBN (Bg)6 & 1.52 & 1.52 & -2.90 & $-16.55 * *$ & $-42.65 * *$ & $-27.42 * *$ & $-13.21 * *$ & $-18.45 * *$ & $-28.33 * *$ \\
\hline ADT 3 x PU 31 & -2.40 & -4.69 & $-11.59 * *$ & $-12.77 * *$ & $-15.06 * *$ & $-23.70 * *$ & 5.00 & 5.00 & $-20.45 * *$ \\
\hline ADT 3 x VBN (Bg)4 & -3.76 & $-7.25 * *$ & $-7.25 * *$ & -2.88 & $-10.11^{*}$ & $-10.11^{*}$ & $-7.76^{*}$ & $-18.94 * *$ & $-18.94 * *$ \\
\hline ADT $3 \times$ VBN $(\mathrm{Bg}) 6$ & -3.08 & -4.55 & $-8.70 * *$ & $17.98 * *$ & -8.16 & $-21.84 * *$ & -6.34 & -7.25 & $-28.33 * *$ \\
\hline CO $5 \times$ PU 31 & 2.29 & -4.29 & -2.90 & $-18.85 * *$ & $-33.24 * *$ & -7.07 & 2.41 & -4.66 & $-16.21 * *$ \\
\hline CO $5 \times$ VBN $(\mathrm{Bg}) 4$ & 0.72 & 0.00 & 1.45 & $-17.84 * *$ & $-29.41 * *$ & -1.74 & $-15.81 * *$ & $-20.91 * *$ & $-20.91 * *$ \\
\hline CO $5 \times$ VBN $(B g) 6$ & 4.41 & 1.43 & 2.90 & $-16.36 * *$ & $-43.94 * *$ & $-21.96 * *$ & -4.04 & $-9.83^{*}$ & $-20.76^{* *}$ \\
\hline CO $6 \times$ PU 31 & -3.28 & -3.28 & $-14.49 * *$ & -3.75 & -4.28 & $-14.02 * *$ & -1.25 & -5.20 & $-28.18 * *$ \\
\hline CO $6 \times$ VBN (Bg)4 & 0.00 & $-5.80 *$ & $-5.80 *$ & $-16.10 * *$ & $-20.78 * *$ & $-20.78 * *$ & $-12.14 * *$ & $-25.45 * *$ & $-25.45^{* *}$ \\
\hline CO $6 \times$ VBN $(\mathrm{Bg}) 6$ & 0.79 & -3.03 & $-7.25 * *$ & 7.01 & $-17.95 * *$ & $-27.11 * *$ & -4.12 & $-8.82 *$ & $-29.55 * *$ \\
\hline LBG $623 \times$ PU 31 & -3.70 & $-12.16 * *$ & $-5.80 *$ & $-22.76 * *$ & $-35.64 * *$ & $-13.28 * *$ & -2.78 & $-9.48 *$ & $-20.45 * *$ \\
\hline LBG 623 x VBN $(B g) 4$ & -3.50 & $-6.76 * *$ & 0.00 & $-21.88 * *$ & $-31.95 * *$ & -8.31 & $-15.16 * *$ & $-20.30 * *$ & $-20.30 * *$ \\
\hline LBG 623 x VBN (Bg)6 & $-5.71 *$ & $-10.81 * *$ & -4.35 & $-23.16^{* *}$ & $-48.07 * *$ & $-30.02 * *$ & -3.49 & $-9.31 *$ & $-20.30 * *$ \\
\hline LBG 645 x PU 31 & 0.78 & -4.41 & $-5.80 *$ & 2.07 & 1.93 & -8.44 & -5.00 & -5.00 & $-28.03 * *$ \\
\hline LBG 645 x VBN (Bg)4 & 0.73 & 0.00 & 0.00 & -1.57 & -6.70 & -6.70 & $-18.28 * *$ & $-28.18 * *$ & $-28.18 * *$ \\
\hline LBG 645 x VBN (Bg)6 & 0.00 & -1.47 & -2.90 & $18.08 * *$ & -9.72 & $-19.13 * *$ & -6.53 & -7.45 & $-28.48 * *$ \\
\hline LBG 685 x PU 31 & -3.82 & $-10.00 * *$ & $-8.70 * *$ & $-10.35^{*}$ & $-11.56^{*}$ & $-18.36 * *$ & $34.40 * *$ & $34.40 * *$ & 1.82 \\
\hline LBG 685 x VBN $(B g) 4$ & -2.16 & -2.86 & -1.45 & $-10.97 * *$ & $-14.39 * *$ & $-14.39 * *$ & $-18.28 * *$ & $-28.18 * *$ & $-28.18 * *$ \\
\hline LBG 685 x VBN (Bg)6 & -1.47 & -4.29 & -2.90 & 4.53 & $-20.90 * *$ & $-26.99 * *$ & 3.76 & 2.75 & $-20.61 * *$ \\
\hline LBG 709 x PU 31 & 4.69 & 0.00 & -2.90 & 0.55 & 0.55 & $-9.68 *$ & $45.00 * *$ & $45.00 * *$ & $9.85^{* *}$ \\
\hline LBG 709 x VBN (Bg)4 & 4.41 & 2.90 & 2.90 & -3.20 & -8.13 & -8.13 & $-20.69 * *$ & $-30.30 * *$ & $-30.30 * *$ \\
\hline LBG 709 x VBN (Bg)6 & 2.26 & 1.49 & -1.45 & 8.50 & $-17.13 * *$ & $-25.56 * *$ & 5.94 & 4.90 & $-18.94 * *$ \\
\hline TMV 1 x PU 31 & 3.17 & 0.00 & $-5.80 *$ & -6.18 & -7.69 & $-14.33 * *$ & 5.20 & 5.20 & $-20.30 * *$ \\
\hline TMV 1 x VBN (Bg)4 & 2.99 & 0.00 & 0.00 & $-13.45 * *$ & $-16.56 * *$ & $-16.56 * *$ & $-19.83 * *$ & $-29.55 * *$ & $-29.55^{* *}$ \\
\hline TMV 1 x VBN (Bg)6 & -0.76 & -1.52 & $-5.80 *$ & 3.19 & $-22.06 * *$ & $-27.67 * *$ & 4.16 & 3.14 & $-20.30 * *$ \\
\hline VBN 5 x PU 31 & -0.79 & -4.55 & $-8.70 * *$ & -1.61 & -6.91 & $-16.38 * *$ & -2.20 & -2.20 & $-25.91 * *$ \\
\hline VBN (Bg)5 x VBN (Bg)4 & 2.22 & 0.00 & 0.00 & -2.62 & $-12.28 * *$ & $-12.28 * *$ & $-15.00 * *$ & $-25.30 * *$ & $-25.30 * *$ \\
\hline VBN (Bg)5 x VBN (Bg)6 & 0.00 & 0.00 & -4.35 & $22.96 * *$ & -2.17 & $-21.59 * *$ & -5.94 & -6.86 & $-28.03 * *$ \\
\hline SE & 0.7614 & 0.8791 & 0.8791 & 1.5365 & 1.7742 & 1.7742 & 0.0926 & 0.1070 & 0.1070 \\
\hline
\end{tabular}

$\mathrm{d}_{\mathrm{i}}=$ Relative heterosis; $\mathrm{d}_{\mathrm{ii}}=$ Heterobeltiosis; $\mathrm{d}_{\mathrm{iii}}=$ Standard heterosis; ** Significant at $1 \%$ level, $*$ Significant at $5 \%$ level 
Table.3 Heterosis percent for Number of clusters/plant, Number of pods/plant and Pod length

\begin{tabular}{|c|c|c|c|c|c|c|c|c|c|}
\hline \multirow[t]{2}{*}{ Crosses } & \multicolumn{3}{|c|}{ Number of clusters/plant } & \multicolumn{3}{|c|}{ Number of pods/plant } & \multicolumn{3}{|c|}{ Pod length } \\
\hline & $\mathbf{d}_{\mathbf{i}}$ & $d_{i i}$ & $\mathbf{d}_{\mathrm{iii}}$ & $d_{i}$ & $\mathbf{d}_{\mathrm{ii}}$ & $\mathbf{d}_{\mathrm{iii}}$ & $\mathbf{d}_{\mathbf{i}}$ & $\mathbf{d}_{\mathrm{ii}}$ & $d_{\text {iii }}$ \\
\hline ACM 05007 x PU 31 & 1.37 & $-15.61 * *$ & $-17.29 * *$ & $24.57 * *$ & $18.06^{* *}$ & 8.36 & 1.32 & 1.10 & $-2.65^{*}$ \\
\hline ACM 05007 x VBN (Bg)4 & -2.41 & $-19.40 * *$ & $-19.40 * *$ & $14.29 * *$ & 4.11 & 4.11 & 1.35 & -0.53 & -0.53 \\
\hline ACM $05007 \times$ VBN(Bg) 6 & $6.67 *$ & $-14.02 * *$ & $-8.46^{*}$ & $25.31 * *$ & $17.94 * *$ & $9.86^{*}$ & -1.37 & $-5.35^{* *}$ & -0.85 \\
\hline MDU 1 x PU 31 & 2.74 & $-14.47 * *$ & $-16.17 * *$ & $26.53 * *$ & $23.13 * *$ & $13.01 * *$ & $3.75 * *$ & 0.66 & $-3.49 * *$ \\
\hline MDU 1x VBN (Bg)4 & 0.00 & $-17.41 * *$ & $-17.41 * *$ & $11.29 * *$ & 3.97 & 3.97 & $4.28 * *$ & -0.85 & -0.85 \\
\hline MDU 1 x VBN (Bg)6 & $-9.57 * *$ & $-27.10 * *$ & $-22.39 * *$ & 5.78 & 2.21 & -4.79 & -0.22 & $-7.17 * *$ & $-2.75^{*}$ \\
\hline ADT 3 x PU 31 & $-11.98 * *$ & $-25.38 * *$ & $-26.87 * *$ & $10.81^{*}$ & 8.66 & -0.27 & $3.57 * *$ & 2.54 & -1.69 \\
\hline ADT $3 \times$ VBN (Bg)4 & -5.77 & $-20.77 * *$ & $-20.77 * *$ & 1.53 & -4.45 & -4.45 & $2.56^{*}$ & -0.53 & -0.53 \\
\hline ADT $3 \times$ VBN (Bg)6 & -2.76 & $-20.26 * *$ & $-15.10 * *$ & -5.06 & -7.57 & $-13.90 * *$ & -2.13 & $-7.17 * *$ & $-2.75^{*}$ \\
\hline CO 5 x PU 31 & $-15.85^{* *}$ & $-21.41 * *$ & $-11.24 * *$ & $14.93 * *$ & 2.24 & -6.16 & -1.03 & $-7.49 * *$ & 2.01 \\
\hline CO 5 x VBN (Bg)4 & $-22.78 * *$ & $-27.20 * *$ & $-17.79 * *$ & 3.67 & $-11.10 *$ & $-11.10 *$ & $-6.19 * *$ & $-10.56 * *$ & -1.38 \\
\hline CO $5 x$ VBN $(B g) 6$ & $-24.60 * *$ & $-26.76 * *$ & $-17.29 * *$ & -3.16 & $-14.41 * *$ & $-20.27 * *$ & $-9.35^{* *}$ & $-11.61 * *$ & $-2.54 *$ \\
\hline CO $6 \times$ PU 31 & -3.17 & $-18.65 * *$ & $-20.27 * *$ & $21.59 * *$ & $17.24 * *$ & 7.60 & 0.33 & -0.76 & $-2.75^{*}$ \\
\hline CO $6 x$ VBN (Bg)4 & $-24.78 * *$ & $-37.31 * *$ & $-37.31 * *$ & $10.87^{*}$ & 2.67 & 2.67 & -1.98 & $-2.96 *$ & $-2.96^{*}$ \\
\hline CO $6 \times$ VBN $(\mathrm{Bg}) 6$ & $-24.71 * *$ & $-38.79 * *$ & $-34.83 * *$ & 7.83 & 3.24 & -3.84 & $-3.65 * *$ & $-6.77 * *$ & -2.33 \\
\hline LBG $623 \times$ PU 31 & $-25.36 * *$ & $-30.00 * *$ & $-21.64 * *$ & 3.96 & -7.91 & $-15.48 * *$ & -1.45 & $-6.96 * *$ & 0.42 \\
\hline LBG $623 \times$ VBN (Bg)4 & $-10.09 * *$ & $-14.89 * *$ & -4.73 & -6.98 & $-20.55^{* *}$ & $-20.55^{* *}$ & $-4.43 * *$ & $-7.94 * *$ & -0.63 \\
\hline LBG $623 \times$ VBN $(\mathrm{Bg}) 6$ & $-27.56^{* *}$ & $-29.33 * *$ & $-20.90 * *$ & 5.60 & -7.06 & $-13.42 * *$ & $-5.37 * *$ & $-6.76^{* *}$ & 0.63 \\
\hline LBG $645 \times$ PU 31 & $-13.04 * *$ & $-18.78 * *$ & $-20.40 * *$ & $20.80 * *$ & $19.39 * *$ & $12.19 * *$ & $2.25 *$ & -0.52 & 0.85 \\
\hline LBG 645 x VBN (Bg)4 & $-30.65 * *$ & $-35.82 * *$ & $-35.82 * *$ & $13.70 * *$ & $10.27 *$ & $10.27 *$ & $6.46 * *$ & $5.74 * *$ & $7.20^{* *}$ \\
\hline LBG 645 x VBN (Bg)6 & $-12.21 * *$ & $-21.03 * *$ & $-15.92 * *$ & $9.37 *$ & 8.89 & 2.33 & $-2.57 *$ & $-4.14 * *$ & 0.42 \\
\hline LBG $685 \times$ PU 31 & $-22.75 * *$ & $-25.89 * *$ & $-27.36^{* *}$ & -3.57 & $-13.43 * *$ & $-20.55 * *$ & -1.59 & $-5.58 * *$ & -1.48 \\
\hline LBG 685 x VBN (Bg)4 & $-33.51 * *$ & $-36.82 * *$ & $-36.82 * *$ & 1.66 & $-12.05 * *$ & $-12.05^{* *}$ & $-2.85^{*}$ & $-4.87 * *$ & -0.74 \\
\hline LBG 685 x VBN $(\mathrm{Bg}) 6$ & $-35.70 * *$ & $-40.65 * *$ & $-36.82 * *$ & -6.76 & $-16.84 * *$ & $-22.53 * *$ & $-5.47 * *$ & $-5.66^{* *}$ & -1.16 \\
\hline LBG 709 x PU 31 & $30.79 * *$ & $21.83 * *$ & $19.40 * *$ & $33.43 * *$ & $32.84 * *$ & $21.92 * *$ & $15.81 * *$ & $14.44 * *$ & $12.38 * *$ \\
\hline LBG 709 x VBN (Bg)4 & $-16.44 * *$ & $-22.89 * *$ & $-22.89 * *$ & 7.25 & 2.40 & 2.40 & $7.10 * *$ & $6.14 * *$ & $6.14 * *$ \\
\hline LBG 709 x VBN (Bg)6 & $-34.38 * *$ & $-41.12 * *$ & $-37.31 * *$ & -2.90 & -4.04 & $-10.62 *$ & $-2.19 *$ & $-5.25^{* *}$ & -0.74 \\
\hline TMV 1 x PU 31 & $-19.35 * *$ & $-31.22 * *$ & $-32.59 * *$ & $-9.56^{*}$ & $-13.88 * *$ & $-20.96 * *$ & -1.30 & $-3.38 * *$ & $-3.28 *$ \\
\hline TMV 1 x VBN (Bg)4 & $-23.38 * *$ & $-35.20 * *$ & $-35.20 * *$ & $-9.81 *$ & $-17.47 * *$ & $-17.47 * *$ & $-7.77 * *$ & $-7.82 * *$ & $-7.72 * *$ \\
\hline TMV 1 x VBN (Bg)6 & $-20.40 * *$ & $-34.35^{* *}$ & $-30.10 * *$ & -2.33 & -7.65 & $-13.97 * *$ & $-2.17 *$ & $-4.34 * *$ & 0.21 \\
\hline VBN 5 x PU 31 & $-34.71 * *$ & $-43.65 * *$ & $-44.78 * *$ & 3.09 & -0.30 & -8.49 & $3.84 * *$ & 1.91 & 1.48 \\
\hline VBN (Bg)5 x VBN (Bg)4 & $-32.41 * *$ & $-42.16 * *$ & $-42.16^{* *}$ & 0.59 & -6.58 & -6.58 & $-7.53 * *$ & $-7.72 * *$ & $-7.72 * *$ \\
\hline VBN (Bg)5 x VBN (Bg)6 & $-35.01 * *$ & $-45.79 * *$ & $-42.29 * *$ & 4.75 & 0.59 & -6.30 & $-9.06 * *$ & $-11.31 * *$ & $-7.09 * *$ \\
\hline SE & 0.5508 & 0.6360 & 0.6360 & 1.3976 & 1.6138 & 1.6138 & 0.0517 & 0.0597 & 0.0597 \\
\hline
\end{tabular}

$\mathrm{d}_{\mathrm{i}}=$ Relative heterosis; $\mathrm{d}_{\mathrm{ii}}=$ Heterobeltiosis; $\mathrm{d}_{\mathrm{iii}}=$ Standard heterosis; ** Significant at $1 \%$ level, * Significant at $5 \%$ level 
Table.4 Heterosis percent for Number of seeds/pod, Hundred seed weight and Seed yield/plant

\begin{tabular}{|c|c|c|c|c|c|c|c|c|c|}
\hline \multirow[t]{2}{*}{ Crosses } & \multicolumn{3}{|c|}{ Number of seeds/pod } & \multicolumn{3}{|c|}{ Hundred seed weight } & \multicolumn{3}{|c|}{ Seed yield/plant } \\
\hline & $d_{i}$ & $\mathbf{d}_{\mathrm{ii}}$ & $\mathbf{d}_{\mathrm{iii}}$ & $\mathbf{d}_{\mathrm{i}}$ & $\mathbf{d}_{\mathrm{ii}}$ & $\mathbf{d}_{\mathrm{iii}}$ & $\mathbf{d}_{\mathrm{i}}$ & $\mathbf{d}_{\mathrm{ii}}$ & $\mathbf{d}_{\mathrm{iii}}$ \\
\hline ACM 05007 x PU 31 & $9.87 * *$ & $8.50 * *$ & 0.15 & 2.23 & 1.29 & $-5.90 * *$ & $20.55 * *$ & 10.94 & -2.23 \\
\hline ACM 05007 x VBN (Bg)4 & $8.00 * *$ & 3.85 & 3.85 & $-4.72 * *$ & $-8.10 * *$ & $-8.10 * *$ & 8.14 & -5.88 & -5.88 \\
\hline ACM 05007 x VBN(Bg) 6 & 3.15 & -2.24 & 0.77 & -1.87 & $-5.13 * *$ & $-5.60 * *$ & $12.17 *$ & -1.07 & -4.08 \\
\hline MDU 1 x PU 31 & $4.53^{*}$ & 0.79 & -2.31 & 0.75 & -1.67 & $-5.80 * *$ & $23.34 * *$ & $14.15^{*}$ & 0.60 \\
\hline MDU 1x VBN (Bg)4 & 2.81 & 1.23 & 1.23 & $-6.13 * *$ & $-8.10 * *$ & $-8.10 * *$ & 5.32 & -7.84 & -7.84 \\
\hline MDU 1 x VBN (Bg)6 & 0.69 & -2.31 & 0.69 & 1.79 & -0.10 & -0.60 & 7.38 & -4.78 & -7.68 \\
\hline ADT 3 x PU 31 & $6.25 * *$ & 3.66 & -1.92 & $-11.98 * *$ & $-21.05 * *$ & $-28.00 * *$ & $14.54 *$ & 4.45 & -7.95 \\
\hline ADT 3 x VBN (Bg)4 & $6.01 * *$ & 3.15 & 3.15 & $-11.02 * *$ & $-23.30 * *$ & $-23.30 * *$ & $23.95 * *$ & 6.97 & 6.97 \\
\hline ADT 3 x VBN (Bg)6 & 2.10 & -2.09 & 0.92 & $-9.95 * *$ & $-22.21 * *$ & $-22.60 * *$ & 5.17 & -8.03 & $-10.84 *$ \\
\hline CO $5 \times$ PU 31 & $5.34^{*}$ & 4.45 & $-4.38 *$ & $16.55^{* *}$ & $3.07 *$ & $-6.00 * *$ & $33.02 * *$ & 3.71 & -8.61 \\
\hline CO $5 \times$ VBN $(\mathrm{Bg}) 4$ & $6.02 * *$ & 1.54 & 1.54 & 2.41 & $-12.90 * *$ & $-12.90 * *$ & $21.56 * *$ & -9.26 & -9.26 \\
\hline CO $5 \times$ VBN $(B g) 6$ & $-10.12 * *$ & $-15.15 * *$ & $-12.54 * *$ & $15.45^{* *}$ & -1.61 & -2.10 & 9.27 & $-17.58 * *$ & $-20.10 * *$ \\
\hline CO $6 \times$ PU 31 & $20.17 * *$ & $18.63^{* *}$ & $6.77 * *$ & $10.45^{* *}$ & $9.54 * *$ & -0.10 & $33.29 * *$ & $21.76^{* *}$ & 7.30 \\
\hline CO $6 \times$ VBN (Bg)4 & $5.82 * *$ & -0.69 & -0.69 & $7.64 * *$ & 2.10 & 2.10 & $30.50 * *$ & $12.80 *$ & $12.80 *$ \\
\hline CO $6 \times$ VBN $(\mathrm{Bg}) 6$ & $7.82 * *$ & -0.22 & 2.85 & $3.59 * *$ & -1.51 & -2.00 & $20.53 * *$ & 5.56 & 2.34 \\
\hline LBG $623 \times$ PU 31 & $11.35^{* *}$ & $8.97 * *$ & -1.92 & $7.77 * *$ & $-5.70 * *$ & $-14.00 * *$ & $20.40 * *$ & -6.98 & $-18.03 * *$ \\
\hline LBG 623 x VBN $(B g) 4$ & -2.48 & $-9.23 * *$ & $-9.23 * *$ & 0.01 & $-15.80 * *$ & $-15.80 * *$ & -5.52 & $-30.07 * *$ & $-30.07 * *$ \\
\hline LBG 623 x VBN (Bg)6 & $-6.67 * *$ & $-14.33 * *$ & $-11.69 * *$ & -0.18 & $-15.78 * *$ & $-16.20 * *$ & 0.75 & $-24.66 * *$ & $-26.96 * *$ \\
\hline LBG 645 x PU 31 & $6.61 * *$ & 4.43 & -2.00 & $3.87 * *$ & 1.90 & $-3.40 *$ & $40.76^{* *}$ & $38.94 * *$ & $22.44 * *$ \\
\hline LBG 645 x VBN (Bg)4 & $6.75 * *$ & 3.46 & 3.46 & -2.05 & $-4.60 * *$ & $-4.60 * *$ & $29.54 * *$ & $20.37 * *$ & $20.37 * *$ \\
\hline LBG 645 x VBN (Bg)6 & $5.78 * *$ & 1.04 & 4.15 & $7.36 * *$ & $4.82 * *$ & $4.30 * *$ & $44.34 * *$ & $36.07 * *$ & $31.92 * *$ \\
\hline LBG 685 x PU 31 & $10.78 * *$ & $8.89 * *$ & -2.00 & $11.26^{* *}$ & 1.86 & $-7.10 * *$ & $23.41 * *$ & -1.61 & $-13.29 *$ \\
\hline LBG 685 x VBN (Bg)4 & $7.98 * *$ & 0.92 & 0.92 & $-6.94 * *$ & $-18.20 * *$ & $-18.20 * *$ & $13.58 *$ & $-13.45^{*}$ & $-13.45^{*}$ \\
\hline LBG 685 x VBN (Bg)6 & $-7.69 * *$ & $-14.93 * *$ & $-12.31 * *$ & -1.08 & $-12.86 * *$ & $-13.30 * *$ & -9.26 & $-30.11 * *$ & $-32.24 * *$ \\
\hline LBG 709 x PU 31 & $12.15 * *$ & $6.54 * *$ & $6.54 * *$ & 2.25 & 0.11 & $-4.70 * *$ & $75.24 * *$ & $72.81 * *$ & $52.29 * *$ \\
\hline LBG 709 x VBN (Bg)4 & 2.69 & 2.69 & 2.69 & $-7.07 * *$ & $-9.30 * *$ & $-9.30 * *$ & $13.46^{* *}$ & 5.34 & 5.34 \\
\hline LBG $709 \times$ VBN (Bg)6 & 1.44 & -0.07 & 3.00 & $-4.98 * *$ & $-7.04 * *$ & $-7.50 * *$ & 0.57 & -5.28 & -8.17 \\
\hline TMV 1 x PU 31 & $4.75^{*}$ & 0.63 & -1.69 & $-6.10 * *$ & $-8.00 * *$ & $-16.10 * *$ & $-16.04 * *$ & $-22.68 * *$ & $-31.86 * *$ \\
\hline TMV 1 x VBN (Bg)4 & -3.74 & $-4.85^{*}$ & $-4.85^{*}$ & $-6.03 * *$ & $-11.90 * *$ & $-11.90 * *$ & -7.44 & $-19.39 * *$ & $-19.39 * *$ \\
\hline TMV 1 x VBN (Bg)6 & $-8.97 * *$ & $-11.34 * *$ & $-8.62 * *$ & $5.03 * *$ & -1.31 & -1.80 & 5.60 & -6.80 & -9.64 \\
\hline VBN 5 x PU 31 & 0.67 & -1.79 & $-7.08 * *$ & $-4.79 * *$ & $-7.35 * *$ & $-15.50 * *$ & 6.01 & -0.87 & $-12.64 *$ \\
\hline VBN (Bg)5 x VBN (Bg)4 & -3.16 & $-5.77 * *$ & $-5.77 * *$ & $-3.60 * *$ & $-10.20 * *$ & $-10.20 * *$ & 4.07 & -8.06 & -8.06 \\
\hline VBN (Bg)5 x VBN (Bg)6 & -2.02 & $-6.04 * *$ & -3.15 & $-8.40 * *$ & $-14.47 * *$ & $-14.90 * *$ & 3.89 & -6.97 & -9.80 \\
\hline SE & 0.1210 & 0.1397 & 0.1397 & 0.0596 & 0.0688 & 0.0688 & 0.4184 & 0.4831 & 0.4831 \\
\hline
\end{tabular}

$\mathrm{d}_{\mathrm{i}}=$ Relative heterosis; $\mathrm{d}_{\mathrm{ii}}=$ Heterobeltiosis; $\mathrm{d}_{\mathrm{iii}}=$ Standard heterosis; $* *$ Significant at $1 \%$ level, * Significant at $5 \%$ level 
In case of plant height trait, 24 inter-varietal crosses showed significant negative standard heterosis whereas none of the hybrids reported for significant positive standard heterosis In general, negative and significant heterosis over standard parent was noted in the inter-varietal crosses for days to $50 \%$ flowering and plant height implying earliness in maturity is the deciding factor in selection of short duration genotypes in different heterotic cross combinations. Similar findings were reported by Gupta (2005) and Kant and Srivastava (2012). Number of branches/plant shows significant positive values in all three format of heterosis. However, only one cross combination alone LBG 709 x PU 31 recorded standard heterosis in significantly positive direction. This result is in conformity with earlier reports of Pandiyan et al., (2010) and Yashpal et al., (2015). Only one heterotic cross combination LBG 709 x PU 31 showed desired higher magnitude of heterosis for cluster/ plant over commercial check indicating the preponderance of dominance for heterotic expression of this trait. Because increase in number of cluster/plant will contribute to increase in number of pods and then on yield. A similar finding on cluster/ plant was reported by Barad et al., (2008), Thomas et al., (2008) and Kant and Srivastava (2012). For the trait number of pods/plant, 12, seven and five crosses registered significant positive relative heterosis, heterobeltiosis and standard heterosis respectively. The cross LBG 709 x PU 31registered the maximum values of $33.43,32.84$ and 21.92 per cent for relative heterosis, heterobeltiosis and standard heterosis respectively for this trait. Positive significant heterosis for pods/plant is desirable for selection of high yielding genotypes from crosses. The result was akin with the findings of Bagade et al., (2002); Patil et al., (2012) in Indian bean. Pod length is one of the important yield attributing trait which decides the seed number and size. For this trait, nine crosses expressed significant and positive relative heterosis while three crosses displayed significantly positive heterobeltiosis and standard heterosis. Similar observation was made by Bagade et al., (2002) and Kant and Srivastava (2012).

For the trait, number of seeds/pod, 17 crosses exhibited significant and positive relative heterosis while five crosses displayed significantly positive heterobeltiosis and two crosses for standard heterosis. Similar result was also reported by Patil et al., (2012) in lab lab bean and Kant and Srivastava (2012) in urdbean. Out of 33 inter-varietal crosses, 10, three and one crosses registered significant positive relative heterosis, heterobeltiosis and standard heterosis respectively for hundred seed weight. With regard to seed yield/plant, positive and significant values were recorded in18, seven and five crosses for relative heterosis, heterobeltiosis and standard heterosis respectively. Estimation of heterosis for yield per plant had also been conducted by Patel et al., (2009), Reddy et al., (2011) and Ram et al., (2013) who reported significant positive heterosis for seed yield/ plant.

It is concluded, based on the results of this investigation, the inter-varietal crosses viz., LBG 709 x PU 31, LBG 645 x VBN (Bg)6 and LBG $645 \times$ PU 31 exhibited high positive significant standard heterosis for seed yield / plant and its yield attributing traits in positive direction and negative heterosis for two traits viz., days to $50 \%$ flowering and plant height. Besides, these crosses registered high standard heterosis of $52.29 \%, 31.92 \%$, and $22.44 \%$ respectively for seed yield /plant.

The existence of magnitude of heterosis was higher in these crosses for yield and its attributes. Therefore, exploitation of hybrid vigour could be achieved in these crosses and might aid in the genetic enhancement of this crop. 


\section{Acknowledgements}

The first author is highly thankful to ICAR for providing Senior Research Fellowship (2014-2015) for PhD study at Tamil Nadu Agricultural University, Coimbatore.

\section{References}

Bagade, A.B., Patel, D.U., Singh, B and Desai, N.C. (2002). Heterosis for yield and yield component in Indian bean (Dolichos lablab L.). Indian Journal of Pulses Research, 15:46-48.

Barad, H.R., Pithia, M.S and Vachhani, J. H. (2008). Heterosis and combining ability studies for economic traits in genetically diverse lines of mungbean (Vigna radiata (L.) Wilczek). Legume Research., 31: 250-3.

Fonseca, S. and Patterson, F.L. (1968) Hybrid vigour in seven parental diallel crosses in common wheat (Triticum aestivum L.). Crop Sciences, 8: 85-8.

Grakh,S.S. and Chaudhary, M.S. (1985). Heterosis for early maturing and high yield in Gossypium arboreum. Indian Journal of Agricultural Sciences, 55:10-13.

Gupta RS. (2005). Genetic study for yield and its component traits in urdbean [Vigna mungo (L) Hepper]. Ph. D. Thesis, NDUA\&T, Faizabad (UP), India.

Hays HK, Immer FR and Smith DC. (1955). Methods of Plant Breeding. McGraw hill Book Co., Inc., New York. Jackson MK. 1976. Soil chemical analysis. Prentice Hall of India Pvt. Ltd., India.

Kant R and Srivastava RK. (2012). Inheritance of some quantitative characters in urdbean [Vigna mungo (L.) Hepper]. Journal of Food Legumes 25: 1-8

Pandiyan, M., Senthil, N., Ramamoorthi, N., Muthiah, A. R., Tomooka, N., Duncan, V and Jayaraj, T. (2010). Interspecific hybridization of Vigna radiate $\times 13$ wild Vigna species for developing MYMV donor. Electronic Journal of Plant Breeding, 1: 600610.

Patel, J. D., Naik, M. R., Chaudhari, S. B., Vaghela, K. O. and Kodappully, V. C. (2009). Heterosis for yield and its components in greengram. Journal of Food Legumes. 22: 256-259.

Patil, A.B., Desai, D.T., Patil, S.A and Patil, S.S. (2012). Heterosis for yield and its components in vegetable lablab bean (Lablab purpureus L). Legume Research, 35: 18-22.

Ram, B., Tikka, S. B. S and Acharya, A. (2013). Heterosis and combining ability in blackgram (Vigna mungo) under different environments. Indian Journal of Agricultural Sciences, 83: 611-616.

Reddy, D. K. R., Venkateswarlu, O., Jyothi, G. L. S and Obaiah, M. C. (2011). Genetic parameters and interrelationship analysis in blackgram [Vigna mungo (L.) Hepper]. Legume Research. 34: 149-152.

Singh, D.P. (2000) Genetics and breeding of black gram and green gram. Govind Ballabh Pant University of Agriculture and Technology, Pantnagar, Uttar Pradesh (now Uttaranchal) Research Bulletin 109:68.

Thomas B., Arya, K., Krishnan Divya and Sreekumar, S. (2008). Heterosis for biological nitrogen fixation traits and yield components in blackgram (Vigna mungo (L.) Hepper). Legume Research, 31: 530-7.

Turner, J.H. (1953). A study of heterosis in upland cotton, combining ability and inbreeding effects. Agronomy Journal, 45: 487-490.

Yashpal, Singh, M.N., Pathak, N and Saroj, S. K. (2015). Combining ability, heterosis and inbreeding depression in inter specific hybrids involving greengram [Vigna radiata (L.) Wilczek] and blackgram [Vigna mungo (L.) Hepper]. Electronic Journal of Plant Breeding, 6: 87-92.

\section{How to cite this article:}

Durga Prasad, A.V.S. and Murugan, E. 2021. Exploitation of Heterosis in Inter-Varietal Crosses of Urdbean (Vigna mungo [L.] Hepper). Int.J.Curr.Microbiol.App.Sci. 10(01): 14051412. doi: https://doi.org/10.20546/ijcmas.2021.1001.167 